\title{
Tumor endothelial cell-derived cadherin-2 promotes angiogenesis and has prognostic significance for lung adenocarcinoma
}

\author{
Huiqin Zhuo ${ }^{1,2^{* \dagger}} \mathbb{D}$, Yan Zhao ${ }^{3+}$, Xiao Cheng ${ }^{4 \dagger}$, Mao Xu ${ }^{1,2+}$, Lin Wang ${ }^{1,2}$, Lingyun Lin ${ }^{1,2}$, Zhi Lyu ${ }^{4}$, Xuehui Hong ${ }^{1,2^{*}}$ and \\ Jianchun $\mathrm{Cai}^{1,2^{*}}$
}

\begin{abstract}
In lung cancer, antiangiogenic strategies targeting tumor-derived endothelial cells (TECs) afford a survival advantage, but the characteristics of TECs have not been comprehensively elucidated. Herein, high-purity (> 98\%) TECs were obtained, and these cells retained expression of EC markers and exhibited high viability. ITRAQ-2DLC-MS/ MS was performed to profile the proteome and the heterogeneity of ECs. Only 31 of 1820 identified proteins were differentially expressed between adenocarcinoma (ADC)- and squamous cell carcinoma (SCC)-derived TECs (TEC-A and TEC-S, respectively), and cadherin-2 (CDH2) was the most significantly upregulated protein in TEC-A samples. Positive immunostaining for $\mathrm{CDH} 2$ (score $>3$ ) was significantly more frequent in the endothelium of ADC tissues than in that of SCC tissues. Loss- or gain-of-function analysis showed that $\mathrm{CDH} 2$ significantly promoted in vitro and in vivo angiogenesis and sensitivity to the antagonist exherin. The MAPK/ERK and MAPKJJNK signaling pathways may play crucial roles in $\mathrm{CDH} 2$-induced HIF-1a/NEGF-mediated angiogenesis. Moreover, high CDH2 expression in TECs was significantly associated with tumor stage, visceral pleural metastasis, and decreased overall survival in patients with ADC but not SCC. Together, these data indicate the importance of $\mathrm{CDH} 2$ in angiogenesis and highlight its potential both for antiangiogenic therapy and as a candidate prognostic marker for ADC.
\end{abstract}

Keywords: Tumor-derived endothelial cell, EC proteome profile, CDH2 expression, Non-small cell lung carcinoma, Heterogeneity

Lung cancer $(\mathrm{LC})$ has long been considered the leading cause of cancer-related deaths worldwide [1]. Many new antiangiogenic strategies (mainly focusing on tumor-derived endothelial cells (TECs)) have been developed, resulting in improved progression-free survival [2]. Therefore, it is necessary to comprehensively elucidate the characteristics of TECs to improve the success of antiangiogenic strategies for LC. Non-small cell lung carcinoma (NSCLC) mostly comprises squamous cell carcinoma (SCC, 28\%) and adenocarcinoma (ADC, $48 \%$ ), which have distinct clinical characteristics, histological presentations, proteomic profiles, metabolic

\footnotetext{
* Correspondence: zhuohuiqin@xmu.edu.cn; hxh5717@163.com; jianchunfh2@sina.com

${ }^{+}$Huiqin Zhuo, Yan Zhao, Xiao Cheng and Mao Xu (These authors contributed equally to this work.)

${ }^{1}$ Department of Gastrointestinal Surgery, The Affiliated Zhongshan Hospital, Xiamen University, Xiamen 361004, Fujian, China

Full list of author information is available at the end of the article
}

phenotypes, immune cell signatures and gene expression subtypes [3-5]. For example, ADC commonly expresses the type II pneumocyte markers SP-C and TIF1; in contrast, SCC preferentially expresses the basal cell markers Trp63 and Krt5/14 [4]. In addition, the expression of glucose transporter GLUT1, is significantly elevated in SCC [5]. The clinical efficacy of currently approved antiangiogenic therapies (i.e., the anti-VEGF inhibitor bevacizumab and the anti-VEGF receptor 2 [VEGFR2] inhibitor ramucirumab) differs between SCC and ADC [6]. However, only a few strategies have been employed to focus on LC-derived TECs because of analytical limitations $[7,8]$, and reports on the heterogeneity of TECs are rare. To our knowledge, large-scale proteomics analysis of ECs in lung ADC, ADC-related TEC-selective proteins, or the heterogeneity of TECs between the $\mathrm{ADC}$ and SCC subtypes has not yet been reported. Therefore, we focused on the mechanism of the most

(c) The Author(s). 2019 Open Access This article is distributed under the terms of the Creative Commons Attribution 4.0 International License (http://creativecommons.org/licenses/by/4.0/), which permits unrestricted use, distribution, and 
important proteins responsible for heterogeneity to facilitate the development of potential therapeutic strategies.

\section{Results/discussion}

EC protein expression profiling based on ITRAQ analysis Primary ECs that were positive for expression of EC markers (CD31, CD34, CD144, CD105, and VWF) but negative for expression of a vascular smooth muscle cell
(VSMC) marker ( $\alpha$-SMA) and monocyte markers $(C D 11 b$ and $C D 45 b)$, with high purity and viability (CD105 expression $>98 \%$, AcLDL uptake $>90 \%$ ), were prepared (Fig. 1; Additional file 1). A total of 1820 proteins were identified. Gene set enrichment analysis of the differentially expressed proteins among ADC-related ECs shown in Additional file 2: Figure S1, including the 81 proteins unique to TECs from ADC (TEC-A)

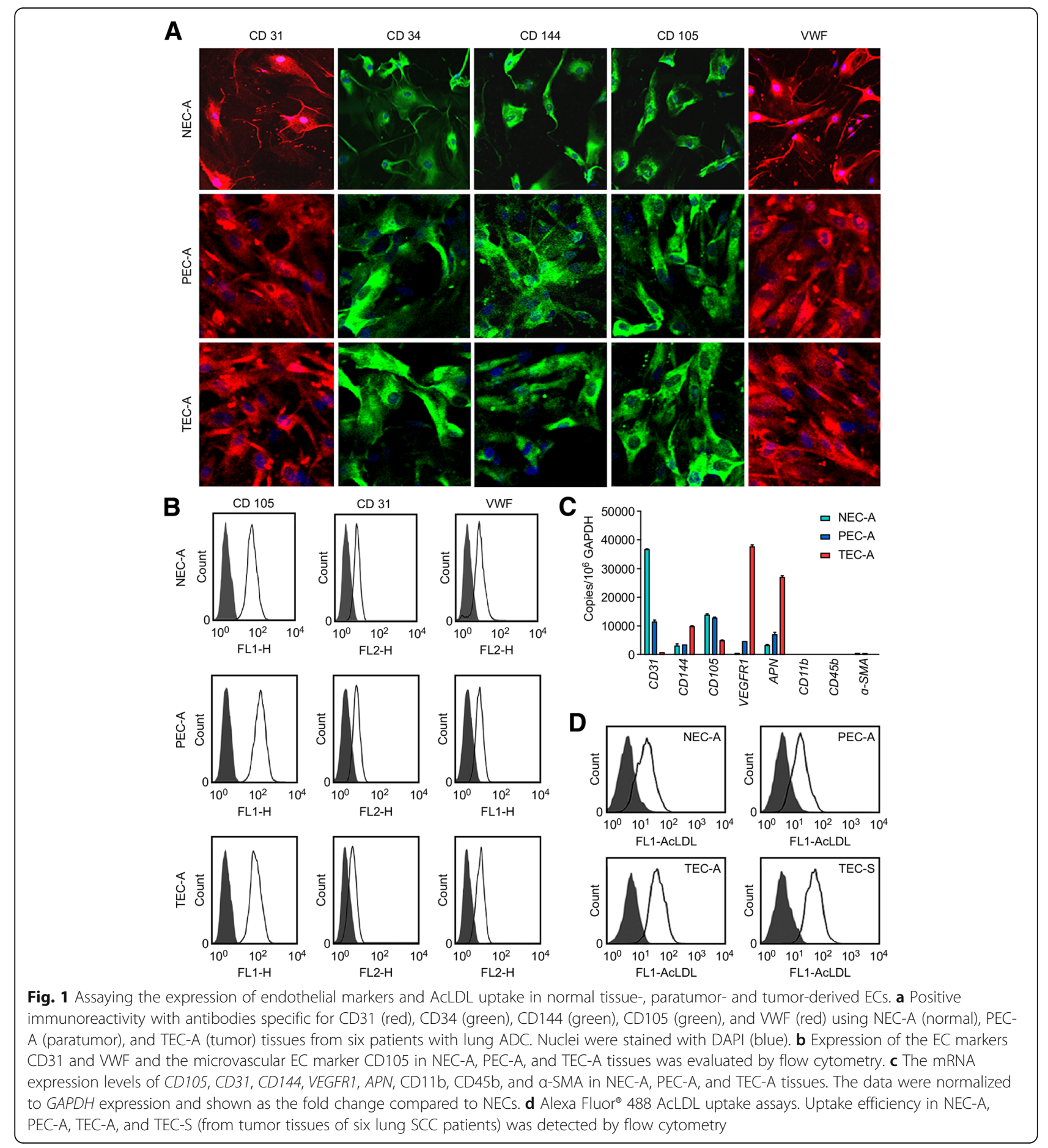









\begin{abstract}
(See figure on previous page.)
Fig. 2 Protein expression patterns and heterogeneity of $\mathrm{CDH} 2$ and Piezo1 expression in ADC- and SCC-derived ECs. a Cluster map comparing the protein expression patterns of NEC-A, PEC-A, TEC-A, and TEC-S. Functional category gene enrichment tests were performed using heatmap.2 and the gregmisc package in the R statistical environment (http://cran.r-project.org/web/packages/gplots). Red indicates higher expression levels, and blue indicates lower expression levels in the two cell types. $\mathbf{b}$ The first and second principle components, PC1 and PC2, from the principle component analysis (PCA). The complete proteomic data sets for the four EC groups were subjected to PCA analysis to capture the maximum variance among ECs, using the SIMCA-P + V12.0.1 software package. The principal components (proteins from the original four groups) were marked with the corresponding name and group color. c Migration assay. The migration of NEC (pool of NEC-A and NEC-S), TEC-S, and TEC-A cells $\left(1.8 \times 10^{4}\right)$ was detected using transwell chambers $(6.5 \mathrm{~mm}, 8.0-\mu \mathrm{m})$, after induction by M131 medium supplemented with $20 \%$ or $3 \%$ MVGS or tumor cell CM at $37^{\circ} \mathrm{C}$ for $12 \mathrm{~h}$. To study EC-induced VSMC migration, cocultivation was established by plating VSMCs $\left(2.4 \times 10^{4}\right)$ onto the filter inserts and transferring to the EC monolayer $\left(5.0 \times 10^{6}\right)$, then incubating in M131 medium supplemented with $0.2 \%$ fetal bovine serum at $37^{\circ} \mathrm{C}$ for $16 \mathrm{~h}$. The cells were stained with $20 \%$ Giemsa solution. The number of migrated cells was counted in five randomly selected fields under a microscope (200x). d Heterogeneity of $\mathrm{CDH} 2$ and Piezo1 expression in ADC- and SCC-derived ECs. Expression of Piezo1 or CDH2 in HUVECs after being cultured in CM-1, CM-2 (SPC-A-1 or L-78 cell-conditioned medium, respectively), Coculture 1 (cocultured with the ADC cell line SPC-A-1), or Coculture 2 (cocultured with the SCC cell line L-78). e Consecutive sections were prepared to detect CD105, CDH2, and Piezo1 expression in the endothelial cells of tumor samples from 68 patients (36 with SCC and 32 with ADC), and CD105 expression was detected as a positive control. The fields shown in dotted lines were enlarged two-fold in the right column, and microvessels are identified using black arrows, based on positive staining for CD105. Expression of Piezo1 or CDH2 in ADC- and SCC-derived ECs, and CDH2 in ECs from tumor tissues (T) and paired paratumor (P) and normal (N) tissues of 32 patients with ADC were statistically analyzed. The intensity of staining (IS) was scored as 0: negative, 1: weak, 2: moderate and 3: strong. The percentage of positive (PP) cells was scored as 0 (PP $\leq 5 \%), 1$ (6\% $\leq$ PP $\leq 25 \%), 2(26 \% \leq P P \leq 50 \%), 3$ $(51 \% \leq \mathrm{PP} \leq 75 \%)$, and 4 (PP $\geq 75 \%)$. The staining in endothelial cells was scored by multiplying the PP by the IS (immunoreactive $s c 0 r e=P P \times I S)$ : 0 (score: $0-2$, negative), $1+$ (score: 3-4, moderately positive), 2++ (score: 5-6, strongly positive), and 3+++ (score: 7-8, very strongly positive)
\end{abstract}

(compared to paratumor-derived ECs from ADC (PEC-A) and normal tissue-derived ECs (NEC-A)), which were mainly involved in transcription activity, protein prepare, hydrogen peroxide catabolic processes, NF- $\mathrm{kB}$ transcription factor activity.

The proteomic profiles were similar in TECs by heatmap analysis (Fig. 2a). A few principal components accounted for $63.3 \%$ ( $\mathrm{PC} 1$ and $\mathrm{PC} 2$ ) of the total data variation, and the locations of the TECs were very close together. Then, the TEC proteomic profiles were analyzed in pairs (Fig. 2b). The results show the proteins (including CDH2, Piezo1, EPS8, NAP1L1, FAM98B, HSPBP1, RPS15 and so on) responsible for segregation into two groups. Only 31 proteins (see Additional file 3: Table S1) were differentially expressed in both groups, among which $\mathrm{CDH} 2$ and Piezo1 were the two most significantly upregulated proteins in the TEC-A group. The expression levels of 15 significantly altered proteins were verified by western blotting (Additional file 4: Figure S2A). Similar changes were observed in PEC-A, TEC-A and tumor cell-EC cocultivation samples. Therefore, all types of isolated ECs provide a good basis for studying tumor angiogenesis and heterogeneity.

\section{Heterogeneity of lung cancer-derived ECs}

EC migration and positive regulation of smooth muscle cells are two important biological processes relevant to angiogenesis. The migration of TECs induced by either M131 medium containing 20\% or 3\% MVGS or tumor cell-conditioned medium (CM) was significantly faster than that of NECs (pool of NEC-A and NEC-S), but TEC-A migration was even faster $(P<0.01)$ than that of TEC-S in the CM group. Moreover, during EC- VSMC cocultivation, although all types of ECs enhanced the migration of VSMCs, TEC-A did so most markedly (Fig. 2c).

The expression levels of Piezo1 and $\mathrm{CDH} 2$ were upregulated in primary human umbilical vein endothelial cells (HUVECs) cocultured with the lung ADC cell line SPC-A-1 but not the SCC cell line L-78 or CM from SPC-A-1 or L-78 cells (Fig. 2d). Positive immunostaining of Piezo1 and $\mathrm{CDH} 2$ (score $>3$ ) was significantly more frequent in TEC-A than in TEC-S samples $(P=0.01$ and $P<0.0001$, respectively; Fig. 2e). In SCC, the expression of $\mathrm{CDH} 2$ was much higher in TECs than in NECs or PECs. Furthermore, the prognostic values revealed that high tumor $\mathrm{CDH} 2$ expression was related to a shorter survival time in LC patients $(P=0.017)$; this correlation was more significant in $\operatorname{ADC}(n=720, P=0.008)$ but not significant in SCC $(n=524, P=0.220)$. However, no similar correlation between Piezo1 expression and survival time was observed (Additional file 4: Figure S2B). These data indicated that $\mathrm{CDH} 2$ likely plays more important functional roles in ADC development than in SCC development.

\section{Effect of $\mathrm{CDH} 2$ on angiogenesis}

Loss- or gain-of-function analysis revealed that $\mathrm{CDH} 2 \mathrm{ex}-$ pression significantly promoted EC proliferation, motility and capillary-like tube formation, whereas silencing endogenous CDH2 had the opposite effect (Additional file 5: Figure S3). A markedly increased density of neovessels (VWF-positive) was observed in Matrigel plugs containing $\mathrm{CDH} 2$-overexpressing ECs (Additional file 6: Figure S4A). Preclinical and clinical data have shown that a peptidic $\mathrm{CDH} 2$ antagonist (exherin, ADH-1) causes rapid tumor vascular disruption and apoptosis [9]. To date, limited research has been conducted on $\mathrm{CDH} 2$-expressing ECs. 

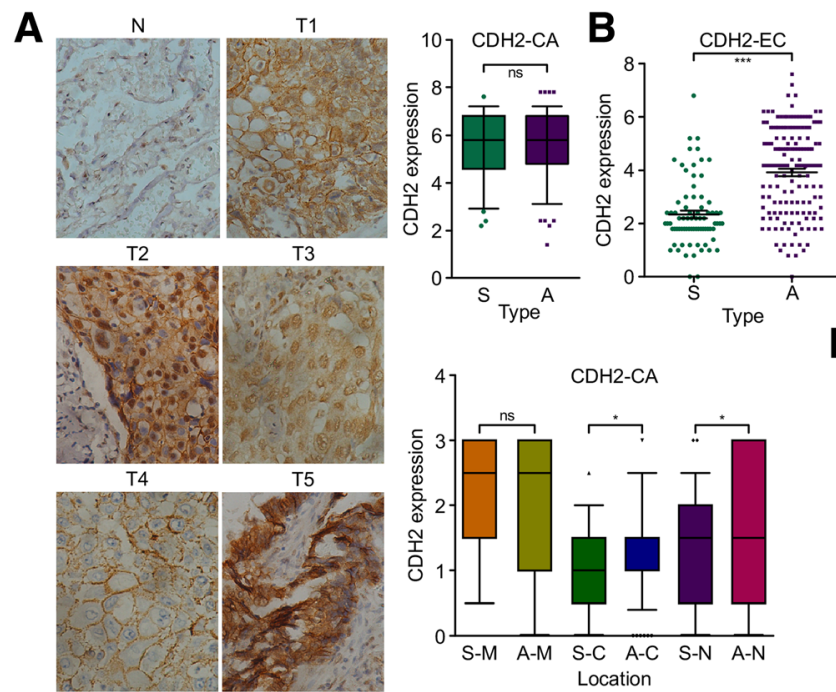

C

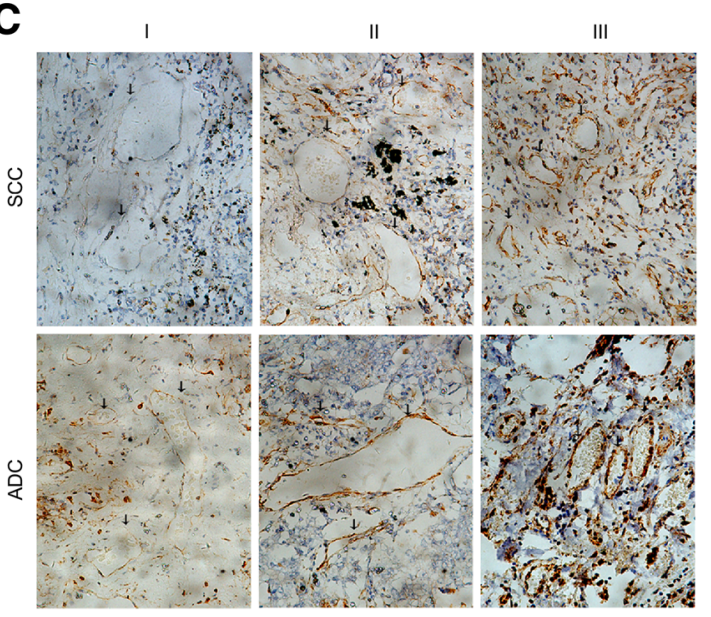

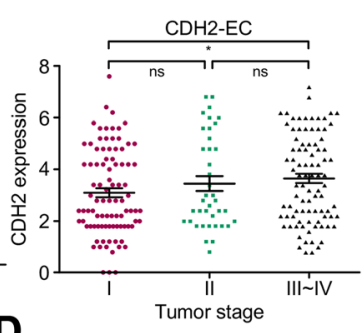
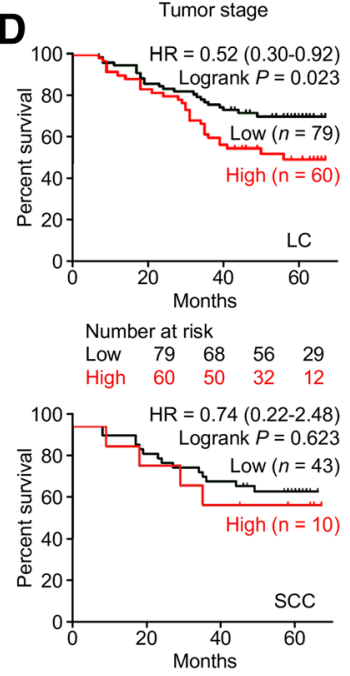

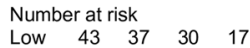

$\begin{array}{lcccc}\text { Low } & 43 & 37 & 30 & 17 \\ \text { High } & 10 & 8 & 6 & 3\end{array}$

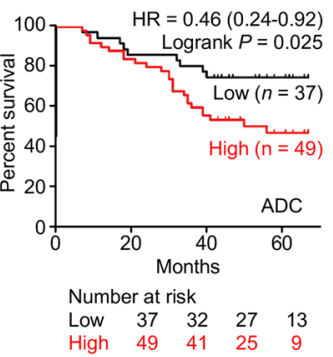

Fig. 3 Aberrantly increased $\mathrm{CDH} 2$ protein expression in ADC-derived ECs by $\mathrm{HC}$ analysis in 218 tumor tissues from lung cancer patients (141 with ADC and 77 with SCC). a $1 \mathrm{HC}$ images of heterogenous $\mathrm{CDH} 2$ localization and $\mathrm{CDH} 2$ expression scores for cancer cells from SCC and ADC samples. IHC staining for SCC and ADC cancer cells was scored by PP $\times I S$. CDH2 expression localization in cancer cells was further rescored by the intensity of staining (IS, score: 0 3) in membrane, cytoplasm and nucleus. b, c High CDH2 expression in TECs from ADC tissues. The significantly positive relationship between $\mathrm{CDH} 2$ expression and tumor stage, as well as typical IHC images displaying increased $\mathrm{CDH} 2$ expression during tumor progression are shown. Microvessels are indicated with black arrow, based on positive staining for CD105. d The overall survival of patients with lung cancer and differential CDH2 expression in TECs. The prognostic value of CDH2 expression was investigated in 139 of 218 patients with lung cancer with respect to survival information

When treated with $0.4 \mathrm{mg} / \mathrm{mL} \mathrm{ADH}-1$ for $24 \mathrm{~h}$, the $\mathrm{CDH} 2$ overexpression group (mainly located in the cell membrane) was significantly inhibited, but this effect was not obvious in the control group. ADH-1 induced apoptosis in a dose- and $\mathrm{CDH} 2$-dependent manner in ECs (Additional file 6: Figure S4B\&C).

\section{Mechanism of $\mathrm{CDH} 2$-mediated angiogenesis}

With $\mathrm{CDH} 2$ overexpression, epithelial cell markers (E-cadherin, EpCAM, and P-cadherin), neural and basal cell adhesion molecules (NCAM-1, NrCAM, and
BCAM), leukocyte rolling and attachment molecules (L-, E-, and P-selectin), and strictly EC-specific adhesion molecules (VE-cadherin) were all downregulated. Moreover, proteins known to positively regulate leukocyte migration (RANTES, ENA-78, IL-6, MCP-2, and IP-1 $\alpha$ ) were also significantly downregulated. Three proteins involved in the VEGFR signaling pathway (VEGFA, VEGFD, and VEGFR3) were significantly upregulated. Furthermore, the phosphorylation levels of two major MAPK signaling pathways, namely, the extracellular signal-regulated kinase (ERK) and c-Jun $\mathrm{N}$-terminal 
kinase (JNK) pathways, were significantly increased, and $\mathrm{CDH} 2$ was confirmed to induce HIF-1 $\alpha$, VEGF, and VEGFR3 accumulation (Additional file 7: Figure S5). Therefore, the MAPK/ERK and MAPK/JNK signaling pathways are likely to play crucial roles in CDH2-induced HIF-1 $\alpha$ /VEGF-mediated angiogenesis.

\section{Clinical value of $\mathrm{CDH} 2$ in $\mathrm{ADC}$}

The adjacent normal tissues were nearly negative for $\mathrm{CDH} 2$ expression, but tumor cells in the lung tissues exhibited very strong positive staining (Fig. 3a, clinical data were listed in Additional file 8: Table S2). The expression of $\mathrm{CDH} 2$ in cancer cells from SCC and ADC did not differ significantly (scored by $\mathrm{PP} \times \mathrm{IS}$ ), but its localization in cancer cells was heterogeneous. Therefore, expression was further rescored by the intensity of staining (IS, score: 0 3) in the membrane, cytoplasm and nucleus. $\mathrm{CDH} 2$ expression in the cytoplasm or nucleus was significantly higher in cancer cells form ADC than in those from SCC $(P<0.05)$. Heterogeneity of $\mathrm{CDH} 2$ cleavage resulted in different regulatory behaviors related to cancer cell-cell adhesion and cell migration/ proliferation [10].

Positive CDH2 staining (score $>3$ ) was seen in 22 and $66 \%$ of TECs from SCC and ADC tumors, respectively (Fig. 3b). High CDH2 expression in TECs was significantly positively related to ADC $(P<0.001)$ and tumor stage $(P<0.05)$ in LC patients, and positively related to tumor stage and visceral pleural metastasis in the ADC subtype $(P<0.05$, Fig. 3b and c; Additional file 9: Table S3). Significantly poorer prognosis and overall survival were associated with patients with high TEC CDH2 expression and LC $(P=0.023)$ or ADC $(n=86$, $P=0.025)$ but not SCC $(n=53, P=0.623)$ (Fig. $3 \mathrm{~d})$. These data suggest that $\mathrm{CDH} 2$ may serve as a prognostic indicator of relative risk for patients with ADC.

\section{Conclusion}

The proteomic profile of ADC-derived ECs was elucidated, and $\mathrm{CDH} 2$, which was uniquely upregulated in TEC-A, was proved to promote in vitro and in vivo angiogenesis and sensitivity to the antagonist ADH-1. The MAPK/ERK and MAPK/JNK signaling pathways may play crucial roles in CDH2-induced HIF-1 $\alpha$ / VEGF-mediated angiogenesis. Strong $\mathrm{CDH} 2$ expression was significantly more frequent in TECs, and this expression pattern was associated with tumor stage, visceral pleural metastasis, and decreased overall survival in patients with ADC but not SCC. These data indicated the important role of $\mathrm{CDH} 2$ in angiogenesis, as well as its potential as both a new molecular target in combination with currently approved anti-angiogenic strategies and a candidate prognostic marker for ADC.

\section{Additional files}

Additional file 1: Materials/Methods. (DOC $46 \mathrm{~kb}$ )

Additional file 2: Figure S1. Functional categorization based on Gene Ontology (GO) and KEGG pathway analysis. Functional categorization of the proteins that were (A) differentially expressed in both the TEC-A and PEC-A groups compared to the NEC-A group, (B) unique TEC-A proteins (compared to the NEC-A and PEC-A groups), and (C) unique PEC-A proteins (compared to the NEC-A and TEC-A groups). Only the top 10 biological processes and KEGG pathways are listed, with statistical significance assigned based on the corrected $P<0.05$. (TIF $10537 \mathrm{~kb}$ )

Additional file 3: Table S1 Proteins differentially expressed in lung adenocarcinoma-derived endothelial cells (TEC-A) in comparison to squamous cell carcinoma-derived endothelial cells (TEC-S). (DOC 68 kb)

Additional file 4: Figure S2. Verification of differential protein expression and the prognostic value of $\mathrm{CDH} 2$ and Piezo 1 in patients with LC, using the Kaplan-Meier plotter database (http://kmplot.com/analysis/ index.php? $p=$ service\&cancer= lung). Lysates from C-A (NEC-A), P-A (PECA), T-A (TEC-A), PC-A (PEC-A cocultured with LTEP- $\alpha-2)$, and TC-A (TEC-A cocultured with LTEP-a-2) cells were prepared from six additional lung ADC patients. Lysates from C-S (NEC-S), T-S (TEC-S), and TC-S (TEC-S cocultured with SK-MES-1) cells were prepared from the six lung SCC patients. The experiments were repeated at least in triplicate. (TIF 6384 kb)

Additional file 5: Figure S3. In vitro assays of $\mathrm{CDH} 2$ function in microvascular endothelial cell (MVEC), Ealy926, and HUVEC lines by overexpressing or knocking down $\mathrm{CDH} 2$ expression. (A) Efficient $\mathrm{CDH} 2$ overexpression or knockdown was confirmed by western blotting. (B, C) Cell survival and apoptosis assays of MEVCs and Ealy926 cells after $\mathrm{CDH} 2$ overexpression or knock down. $(D, E)$ Cell migration and in vitro angiogenic activity of MVECs, Ealy926 cells and HUVECs following $\mathrm{CDH} 2$ overexpression or knock down. Cell numbers were counted in five randomly selected fields under a microscope. (TIF $13045 \mathrm{~kb}$ )

Additional file 6: Figure S4. Matrigel plug assay and ADH-1-induced apoptosis in $\mathrm{CDH}$ 2-expressing cells. (A) Matrigel plug assay. Growth factor-reduced Matrigel matrix (0.25 MI) supplemented with $8.0 \times 10^{5}$ MVECs or Ealy926 cells (mock cells or clones stably expressing $\mathrm{CDH} 2$ ) was injected subcutaneously at the abdominal midline of six-week-old BALB/C nude male mice. Four mice were included in each group. After 10 days, the Matrigel plugs were split and sectioned for anti-WWF (red) and DAPI (blue) staining. The results from two randomly selected models are shown. ( $\mathrm{B}, \mathrm{C}) \mathrm{ADH}-1$-induced apoptosis in $\mathrm{CDH}$-expressing cells, as determined by in vitro assays. $\mathrm{ADH}-1(0,0.1,0.2$, or $0.4 \mathrm{mg} / \mathrm{mL})$ was incubated with control and $\mathrm{CDH}$-overexpressing ED-25 cells for $24 \mathrm{~h}$ (confocal microscopy-based observations) or $48 \mathrm{~h}$ (FCA). Cells incubated with anti-CDH2 (green) and VE-cadherin antibodies (red) were observed with a confocal microscope. Apoptosis was detected using the Annexin V-FITC/PI Kit. (TIF 10193 kb)

Additional file 7: Figure S5. Mechanism of $\mathrm{CDH} 2$-mediated angiogenesis. Angiogenesis antibody (A) and molecular adhesion array (B). HUVECs were infected with an adenoviral vector directing the expression of $\mathrm{CDH} 2$ or a mock vector. Subsequently, the HUVECs were harvested and analyzed for protein expression using RayBiotech Human Angiogenesis Antibody Array C Series 1000 and Adhesion Molecule Array Q1. The blots were scanned with an InnoScan 300 Microarray Scanner and analyzed using ImageJ software. (C) $\mathrm{CDH} 2$ induced upregulation of VEGFA, VEGFR3, MMP-1, and HIF-1a in MVECs and ED-25 cells, as determined by western blotting. Activation of two major MAPK signaling pathways, namely, extracellular signal-regulated kinase (ERK) and c-Jun N-terminal kinase (JNK), was detected. The levels of phosphorylated ERK1/2, JNK, and c-Jun were detected in MVECs and ED-25 cells overexpressing CDH2. (TIF 7116 kb)

Additional file 8: Table S2. Clinical data for 218 lung carcinoma specimens examined in this study. (DOC $274 \mathrm{~kb}$ ) 
Additional file 9: Table S3. Expression of $\mathrm{CDH} 2$ in tumor-derived endothelial cell and its correlation with clinicopathological features of patients with NSCLC. (DOC $82 \mathrm{~kb})$

\section{Abbreviations}

AcLDL: Acetylated low-density lipoprotein; ADC: Adenocarcinoma; BP: Biological processes; $\mathrm{CDH2}$ : Cadherin-2; CM: Cell-conditioned medium; ECs: Endothelial cells; GO: Gene Ontology; LC: Lung cancer; MVGS: Microvascular growth supplement; NSCLC: Non-small cell lung carcinoma; PCA: Principal component analysis; SCC: Squamous cell carcinoma; TECs: Tumor endothelial cells:; VEGF: Vascular endothelial growth factor

\section{Acknowledgements}

Not applicable.

\section{Funding}

This work was supported, in part, by grants from the National Natural Scientific Foundation of China (grant number 81370048), the Natural Science Foundation of Fujian Province (grant numbers 2016 J01631, 2016-ZQN-88, 2016 J01618 and 2017 J01380), and the Projects for Technology Plan of Xiamen in China (grant number 3502Z20174076).

\section{Availability of data and materials}

All data from our study are available upon request.

\section{Authors' contributions}

$\mathrm{HZ}$ conceived of the study, drafted the manuscript and carried out the proteome studies. YZ carried out the preparation of clinical samples and participated in the immunoassays. XC carried out the cell biology studies and immunoassays. MX carried out the animal experiments and participated in the molecular genetic studies. LW performed the statistical analysis and participated in the preparation of clinical samples. LL carried out the cell biology studies and participated in the immunoassays. ZL participated in the proteomic analysis. XH participated in its design and revise. JC participated in its design, revise and coordination. All authors read and approved the final manuscript.

\section{Ethics approval and consent to participate}

All aspects of this study were approved by the medical ethics committee of The Affiliated Zhongshan Hospital of Xiamen University. All tissues used in the study were requested following approval by the Institutional Review Board of the Tumor Tissue Bank of Zhongshan Hospital, Xiamen University. Written informed consent was obtained from all enrolled patients, and all relevant investigations were performed according to the principles of the Declaration of Helsinki. All animal studies were performed with approval from the Xiamen University Laboratory Animal Center.

\section{Consent for publication}

Consent to publish has been obtained from all authors.

\section{Competing interests}

The authors declare that they have no competing interests.

\section{Publisher's Note}

Springer Nature remains neutral with regard to jurisdictional claims in published maps and institutional affiliations.

\section{Author details}

'Department of Gastrointestinal Surgery, The Affiliated Zhongshan Hospital, Xiamen University, Xiamen 361004, Fujian, China. Institute of Gastrointestinal Oncology, School of Medicine, Xiamen University, Xiamen 361004, Fujian, China. ${ }^{3}$ Central Laboratory, The First Hospital Affiliated to Xiamen University, Xiamen University, Xiamen 361004, Fujian, China. ${ }^{4}$ Respiratory Department, The Affiliated Zhongshan Hospital, Xiamen University, Xiamen 361004, Fujian, China.
Received: 29 August 2018 Accepted: 26 February 2019

Published online: 04 March 2019

\section{References}

1. Bray F, Ferlay J, Soerjomataram I, Siegel RL, Torre LA, Jemal A. Global cancer statistics 2018: GLOBOCAN estimates of incidence and mortality worldwide for 36 cancers in 185 countries. CA Cancer J Clin. 2018;68:394-424.

2. Liu YR, Guan YY, Luan X, Lu Q, Wang C, Liu HJ, Gao YG, Yang SC, Dong X, Chen $\mathrm{HZ}$, Fang $\mathrm{C}$. Delta-like ligand 4-targeted nanomedicine for antiangiogenic cancer therapy. Biomaterials. 2015;42:161-71.

3. Zhang W, Wei Y, Ignatchenko V, Li L, Sakashita S, Pham NA, Taylor P, Tsao MS, Kislinger T, Moran MF. Proteomic profiles of human lung adeno and squamous cell carcinoma using super-SILAC and label-free quantification approaches. Proteomics. 2014;14:795-803.

4. Faruki H, Mayhew GM, Serody JS, Hayes DN, Perou CM, Lai-Goldman M. Lung adenocarcinoma and squamous cell carcinoma gene expression subtypes demonstrate significant differences in tumor immune landscape. J Thorac Oncol. 2017;12:943-53.

5. Goodwin J, Neugent ML, Lee SY, Choe JH, Choi H, Jenkins DMR, Ruthenborg RJ, Robinson MW, Jeong JY, Wake M, Abe H, Takeda N, Endo H, Inoue M, Xuan Z, Yoo H, Chen M, Ahn JM, Minna JD, Helke KL, Singh PK, Shackelford DB. Kim JWe. The distinct metabolic phenotype of lung squamous cell carcinoma defines selective vulnerability to glycolytic inhibition. Nat Commun. 2017:8:15503.

6. Chu BF, Otterson GA. Incorporation of antiangiogenic therapy into the nonsmall-cell lung cancer paradigm. Clin Lung Cancer. 2016:17:493-506.

7. Durr E, Yu J, Krasinska KM, Carver LA, Yates JR, Testa JE, Oh P, Schnitzer JE. Direct proteomic mapping of the lung microvascular endothelial cell surface in vivo and cell culture. Nat Biotechnol. 2004;22:985-92.

8. Zhuo H, Lyu Z, Su J, He J, Pei Y, Cheng X, Zhou N, Lu X, Zhou S, Zhao Y. Effect of lung squamous cell carcinoma tumor microenvironment on the CD105+ endothelial cell proteome. J Proteome Res. 2014;13:4717-29.

9. Perotti A, Sessa C, Mancuso A, Noberasco C, Cresta S, Locatelli A, Carcangiu ML, Passera K, Braghetti A, Scaramuzza D, Zanaboni F, Fasolo A, Capri G, Miani M, Peters WP, Gianni L. Clinical and pharmacological phase I evaluation of Exherin $(\mathrm{ADH}-1)$, a selective anti-N-cadherin peptide in patients with $\mathrm{N}$-cadherinexpressing solid tumours. Ann Oncol. 2009;20:741-5.

10. Reiss K, Maretzky T, Ludwig A, Tousseyn T, de Strooper B, Hartmann D, Saftig P. ADAM10 cleavage of $\mathrm{N}$-cadherin and regulation of cell-cell adhesion and beta-catenin nuclear signalling. EMBO J. 2005;24:742-52.

\section{Ready to submit your research? Choose BMC and benefit from:}

- fast, convenient online submission

- thorough peer review by experienced researchers in your field

- rapid publication on acceptance

- support for research data, including large and complex data types

- gold Open Access which fosters wider collaboration and increased citations

- maximum visibility for your research: over $100 \mathrm{M}$ website views per year

At BMC, research is always in progress.

Learn more biomedcentral.com/submissions 